Bioscientia Medicina: Journal of Biomedicine \&

Translational Research

Journal Homepage: www.bioscmed.com

\title{
Klippel Trenaunay Syndrome: A Brief Overview
}

\section{Pande Ayu Naya Kasih Permatananda ${ }^{1 *}$, I Gusti Agung Made Adnyana Putra ${ }^{2}$}

${ }^{1}$ Department of Pharmacology and Pharmacy, Faculty of Medicine and Health Science, Universitas Warmadewa, Denpasar 2Department of Cardiothoracic and Vascular Surgery, Faculty of Medicine, Universitas Airlangga, Surabaya

\section{A R T I C L E I N F O Keywords: \\ Klippel-trenaunay syndrome \\ Capillary malformation \\ Venous varicosities \\ Bone hypertrophy \\ Congenital vascular disease}

\section{*Corresponding author:}

Pande Ayu Naya Kasih Permatananda

E-mail address:

nayakasih@gmail.com

All authors have reviewed and approved the final version of the manuscript.

\section{A B S T R A C T}

Klippel-Trenaunay syndrome is a rare congenital vascular disease. The pathogenesis of this syndrome is unclear, but it is thought that most cases are the result of somatic mutations that affect genes that play a role in vasculogenesis and angiogenesis. Some patients come with a triad of capillary malformation (hemangioma or port-wine stain), venous varicosities and bony or soft tissue hypertrophy. Clinical presentation of this syndrome can lead to significant morbidities and mortalities due to severe bleeding and emboli. Although the number of cases is low, a doctor must be able to distinguish Klippel-Trenaunay Syndrome from other rare vascular disorders. Parkes Weber syndrome is usually similar to Klippel-Trenaunay syndrome, except in the arterial malformations associated with capillary malformations and soft tissue to skeletal or bone hypertrophy. The diagnosis of Klippel-Trenaunay Syndrome is carried out clinically and is quite difficult to do even with experienced doctors because there is no precise pathognomonic test. There are several options in relation to the management of Klippel-Trenaunay Syndrome and non-invasive procedure is considered to be the most important of therapy modalities. Early diagnoses, progression monitoring, and proper intervention should be carried out for better prognosis and preventing complication.

\section{Introduction}

Klippel-Trenaunay Syndrome (KTS) is a complex congenital malformation that affects both the upper and lower extremities, and although rarely can affect the body, neck, or head. There are three important components of Klippel-Trenaunay syndrome, namely varicosities and venous malformations, capillary malformations (port-wine stains), and bone and soft tissue hypertrophy. KTS is a rare disease and mostly occurs sporadically. Its incidence in the world ranges from 2-5 per 100.000. The diagnosis of KTS is carried out clinically and is quite difficult to do even with experienced doctors because there is no precise pathognomonic test. Nonetheless, increased D-dimers and the AGGF1 mutation can help confirm the diagnosis. Duplex ultrasounds, MRIs, and CT scans can show hypertrophy of soft tissue and malformed blood vessels ${ }^{1}$.

Patients with KST are treated similarly to those with other chronic venous disorders. The role of endovascular therapy and minimally invasive interventions has not been widely used because of the small prevalence of this disease, but it is an option compared to open surgery when non-operative management is refractory ${ }^{2}$. This paper would be comprehensively discussing Klippel-Trenaunay Syndrome, especially related to its management in the field of vascular surgery.

\section{Definition}


Klippel-Trenaunay syndrome (KTS) is a complex congenital malformation that affects both the upper and lower extremities, and although rarely can affect the body, neck, or head. There are three important components of Klippel-Trenaunay syndrome, namely varicosities and venous malformations, capillary malformations (port-wine stains), and bone and soft tissue hypertrophy 3

A case of limb hypertrophy was reported in the 19th century, but the combination of congenital vascular nevus in one limb, venous varices in the affected limb, and limb hypertrophy were unrecognized as a unique and consistent syndrome until an article was published by Klippel and Trenaunay in 1900. Several years after that, Frederick Parkes Weber issued a report with a similar case of enlarged arteries and veins, rather than just a venous abnormality. Thus, patients with limb hypertrophy, skin capillary malformations, and arterial and venous malformations are usually diagnosed as Klippel-Trenaunay-Weber syndrome. Parkes Weber syndrome is usually similar to Klippel-Trenaunay syndrome, except in the arterial malformations associated with capillary malformations and soft tissue to skeletal or bone hypertrophy. Meanwhile, SturgeWeber syndrome is defined by the presence of meningeal angioma, capillary malformations of the face, and glaucoma, which is also often accompanied by hemiparesis and hemiatrophy contralateral to meningeal angioma ${ }^{4}$.

Hemangioma in KTS patients is a form of vascular malformation. Anomalies in the deep venous system, such as agenesis, hypoplasia, atresia, valvular incompetence, or venous compression are classic presentations of KTS. Pure hemangioma, which is a proliferation of neoplasms in the endothelium, rarely occurs in KTS, and venous or capillary malformations that occur in KTS contain a static endothelium. An obvious differentiator in KTS is the absence of significant arteriovenous shunting. The presence of arteriovenous shunting of high-flow is the characteristic of Parkes Weber syndrome. In Parkes Weber syndrome, the malformations that occur have significant hemodynamic complications ${ }^{3}$.
Some authors use the term Klippel-TrenaunayWeber syndrome to define a condition that results in an individual or patient having clinically significant arteriovenous malformations as a component of KTS. However, other authors have separated the two conditions and used the term Parkes Weber syndrome for patients who have had arterial malformations in addition to KTS5.

\section{Epidemiology and etiology}

Most cases of KTS occur sporadically. This syndrome has no racial predilection. Parkes Weber syndrome is more common than KTS. Vascular malformations of the skin surface can be seen from birth, but venous varicosity and limb hypertrophy are usually not seen early on. Children were detected in health facilities on average at 4 years of age ${ }^{5-6}$.

Both KTS and Parkes Weber syndrome has a mortality rate of $1 \%$. All patients usually have overt morbidity. Hypertrophy of the extremities or fingers can be extreme, even requiring amputation. Although superficial venous abnormalities are more common, deep veins are usually defective and thrombosis may occur. Pulmonary embolism occurs in $10 \%$ of patients, especially after surgery. (Douma et al., 2012) KTS affects more men than women with an incidence ranging from 2-5 per 100,000 . The pathogenesis of this syndrome is unclear, but it is thought that most cases are the result of somatic mutations that affect genes that play a role in vasculogenesis and angiogenesis ${ }^{4}$.

\section{Pathophysiology}

The causes of KTS can almost be determined, although only with a few theories. Intrauterine damage to the sympathetic ganglia or intermediolateral tract were suggested to be resulted in a microscopic enlargement of the arteriovenous anastomosis. Meanwhile, some literature stated that abnormalities in deep veins result in obstruction of the venous flow resulting in venous hypertension, the appearance of varices, and limb hypertrophy. Mesodermal defects during fetal development also result in impaired microscopic arterial communication. Finally, 
mesodermal and ectodermal dysplasia is considered to be responsible for the appearance of KTS7

Most cases of KST occur sporadically, although some cases in some literature display an autosomal dominant pattern of inheritance 5 . A case report of KTS in monozygous twins with one unaffected twin, supporting the theory of the paradominant pattern of inheritance. This theory states that KTS originates from a single-gene lethal defect in individuals who are homozygous for this gene. Heterozygous carries the gene but is not affected. This disease manifests in individuals who lose heterozygosity through somatic mutations during embryogenesis ${ }^{8}$. There is a significant relationship between angiogenic factors such as AGGF1 and KTS. Kihiczak et al reported that KTS is the result of a pathogenic gene in tissue and vascular development 9 .

KTS is characterized by a combination of vascular malformations of the skin, abnormalities in the lymphatic and venous systems, and enlargement of the limbs. An abnormal venous system results in protrusion of veins on the skin surface, it is called venous flares. Venous varices begin to appear in early childhood and venous anomalies are seen in $80 \%$ of $\operatorname{cases}^{6}$.

Limb hypertrophy occurs due to vascular, venous, and lymphatic abnormalities accompanied by hypertrophy of soft tissues and bones. The hypertrophy that occurs is usually asymmetrical and often affects the fingers. 95\% of cases affected are in the lower extremities. Because vascular abnormalities in KST are not associated with arterial malformations, blood flow to the malformed vessels is usually slow. The combination of slow blood flow to the blood vessels and lymphatic involvement causes the skin lesions to tend to be bluish or purplish. This is different in Parkes Weber syndrome which is characterized by the presence of an arterial fistula, and strong blood flow, resulting in a pink-colored lesion. Cardiac hypertrophy or congestive heart failure is common 6 .

\section{Clinical manifestation}

KTS has a clear clinical spectrum. Ranging from mild port wine stains, varicose veins causing cosmetic deformities to severe disability associated with uncontrolled development of the limbs, chronic pain, clinical infection, arthritis, thromboembolism, and recurrent rectal bleeding due to venous malformations 10. From a study by Noel et al, it was found that $80 \%$ of KTS patients complained of pain, $75 \%$ edema, $40 \%$ bleeding, $15 \%$ superficial thrombophlebitis, and $15 \%$ complained of cellulitis ${ }^{3}$. Most patients show three symptoms of the clinical syndrome, and hemangioma symptoms are the first to occur. Port-wine stain or flat hemangioma is a visible vascular malformation at birth, usually unilateral and segmented, rarely crosses the midline of the body, lined irregularly and firmly. This hemangioma increases with the growth and development of the child and can affect the entire body, although the face and cervix are most frequently affected areas. The lesions may be bright pink in infancy and progressively darken with age. Hemangiomas can be limited or spread to deeper areas of the skin, including the skin, muscles, to the organs, which worsens the prognosis of the disease ${ }^{11}$.

Varicose veins observed in patients with this syndrome are often seen in infancy, but increasingly clear and develop in adulthood. These varicose veins are usually large in the lateral veins, which start from the leg to the leg and radiate proximally to the buttocks or gluteus region. This area can remain stable or enlarge slowly, which eventually results in pain, lymphedema, thrombophlebitis, and ulceration ${ }^{11}$. Deep vein anomalies such as venous hypoplasia, real aneurysms, valve hypoplasia to avalvulia with lymphatic malformations 10 .

Hypertrophy is the third symptom that also appears in this syndrome and can occur secondary to additional length (bone involvement) or increased leg circumference (role of soft tissue influence). It is visible from birth and develops during the first year of life. Towards adolescence, when the child's growth and development have stopped, their growth will also stop $^{11}$. Abnormalities in the bone can affect all of the bones at one extremity or only one or two bones. It 
affects only one leg in about $80-85 \%$ of patients. Not only hypertrophy, but can also be in the form of bone hypotrophy, or other deformities such as macrodactyly, syndactyly, split hand deformity, phalangeal agenesis, and dislocation of the hip joint ${ }^{10}$.

The complications that arise are usually associated with pathological vascular conditions. These complications include static dermatitis, thrombophlebitis, cellulitis, and serious sequelae such as thrombosis, coagulopathy, pulmonary embolism, congestive heart failure (in patients with arteriovenous malformations), and bleeding in the gastrointestinal tract, kidneys, and genitalia. Vascular malformations involving the gastrointestinal tract and urinary tract have been frequently reported and are a significant source of morbidity and mortality. Patients with vascular malformations in the bladder are usually associated with malformations of other organs such as the rectosigmoid and other pelvic organs ${ }^{12}$.

Rectal bleeding and bladder are complications due to pelvic vascular malformations and are reported by 1\%. Gastrointestinal influences are more common in KTS and are believed to occur in as many as $20 \%$ of cases. The locations of the organs most commonly reported to be affected are the colon and rectum. The clinical manifestations range from light bleeding to massive, life-threatening bleeding and coagulopathy. As for genitourinary symptoms, it usually occurs in severe cases. The absence of leg varicose veins does not mean that the pelvic organs are not affected. Vascular malformations of the intraabdominal and intrapelvic organs usually occur in the lower abdomen, pelvis, and skin on the external genitalia. Massive hematuria is often the first symptom of an impact on the bladder and usually occurs early in birth. Vascular malformations of the bladder usually occur in the anterior wall, trigone, and the neck region of the bladder is rarely affected. Some KTS patients also sometimes complain of erectile dysfunction due to abnormalities in the veins in the penis ${ }^{5}$.

\section{Diagnosis}

The diagnosis of KTS is done clinically and is quite difficult to do even with experienced doctors because there is no precise pathognomonic test. Nonetheless, increased D-dimers and the AGGF 1 mutation can help confirm the diagnosis. Duplex ultrasounds, MRIs, and CT scans can show hypertrophy of soft tissue and malformed blood vessels. Plain radiographs can be used to screen long bones for limb length discrepancies. Patients with KTS should first be evaluated using imaging non-invasive diagnostic, the aim of which is to determine the extensiveness and severity of the disease following arteriovenous shunting 1 .

The sensitivity and specificity of duplex ultrasound imaging of the lower extremities are quite high given the anatomy and function of the arteries and veins making this tool the best initial diagnostic step. Confirmation can easily be obtained from the presence of valvular incompetence, low-region area, compressible vascular channels, and anatomic abnormalities, such as aneurysm and atretic segments, which is fast, inexpensive, and does not require sedation, especially in pediatric patients. Hemodynamic studies such as ankle-brachial indices, segmental pressure, and pulse volume recordings can help rule out arterial anomalies ${ }^{13}$.

Most doctors prefer MRI as a diagnostic evaluation in patients with KTS because this imaging technique can provide a better image of soft tissue. This modality can provide the first test for abnormalities in muscles, joints, and bones. In addition, contrast agents such as intravenous gadolinium can differentiate veins from hidden arterial malformations and aid in patient classification in the Hamburg system. MRI and magnetic resonance arteriography (MRA) provide information regarding the extent of vascular lesions such as deep-seated pelvic or thoracic vascular lesions. MRI of the pelvis or thorax is indicated if the venous abnormality is wider than the length of the limb 1 .

CT scan can generate radiation doses that have the potential to cause toxicity to the kidneys, depending on the patient's renal function and the contrast material used. This modality does not provide a detailed picture 
of the muscles and surrounding soft tissue. Therefore, MRI is preferable. In addition, most of the patients undergoing the evaluation for KTS are likely to be children, so avoiding radiation hazards is more important. Venography can be performed to help plan preoperatively when a vein elimination procedure is performed by ablation, sclerotherapy, ligation, or stripping. If documentation of a patent deep venous system has not been worked out beforehand, venography could be used 1 .

Lymphoscintigraphy can be indicated in children with significant leg asymmetry to examine the lymphatic system and the risk of infection 6 . In cases of limb length discrepancy, serial radiographs are required but are not limited to scanogram, and CT scan can be used to measure leg length if necessary. Clinical measurements can only guarantee a measurement in the range of $0.5-1.0 \mathrm{~cm}$, whereas radiological examination can detect differences within $0.1 \mathrm{~cm}$. This check is needed to ascertain how fast the limb is growing and can be used to determine the correct timing for the leg length equalization procedure ${ }^{7}$.

\section{Management}

\section{a. Non-operative management}

Non-operative management is an important modality in the therapy of symptomatic KTS. Usually, only patients who are refractory to drugs undergo surgical intervention. For example, mentioned by Sung et al., Out of 19 KTS patients, only 4 underwent surgery. Patients with KTS are generally treated similarly to patients with other chronic venous disorders ${ }^{14}$.

The initial therapy for patients with chronic vein disease is compression and elevation, which are the basis of leg swelling therapy. Compression stockings should cover the entire swelling area down to the radius and be of appropriate size. The initial pressure can vary, averaging 20-40 mmHg. However, patient compliance with this is quite difficult, especially in children with fast limb growth followed by other social factors. Low-dose aspirin can be considered to be given, although it does not always treat pain and swelling in patients with vascular malformations. If symptoms become refractory to compression, intermittent pneumatic compression can help mobilize lower limb edema. Some clinicians use diuretics in patients with persistent edema despite compression, mobilization, and elevation, although without supporting scientific evidence. If soft tissue inflammation occurs, analgesics can be given, and elevation can also be done, it is necessary to consider antibiotics if cellulitis occurs. There is no evidence of prophylactic antibiotics. The use of anticoagulants is not necessary at the beginning of therapy but should be given if the patient has deep vein thrombosis. Women with venous malformations should avoid using oral contraceptives because of their increased thromboembolic risk ${ }^{1,15}$.

Limb length discrepancies can be treated by giving heels or special shoes to treat scoliosis if the gap is less than $1.5 \mathrm{~cm}$. However, if the gap is more than $2 \mathrm{~cm}$, intervention in the form of osteotomy or epiphysiodesis should be done. Using the Clinical-EtiologicalAnatomical-Pathophysiological (CEAP) classification, vascular intervention should be considered when disease progression is more than class 3 or class 2 with symptoms. Absolute indications for practical intervention consisted of continuous bleeding, refractory ulceration, and acute thromboembolism. While the relative indications consist of pain, functional disorders, edema due to venous insufficiency, leg asymmetry, and $\operatorname{cosmetics}^{1}$. The presentation of various cases and disease severity means that each action must be suitable for each patient. A multidisciplinary team must be involved in patient care. Patients should also be educated about the disease to increase adherence to therapy ${ }^{16}$.

\section{b. Semi-invasive management}

The role of endovascular therapy and minimally invasive interventions has not been widely practiced because the prevalence of this disease is still small, but it is an option compared to open surgery when nonoperative management is refractory. The success of sclerotherapy, embolism, ablation, and laser therapy in voluntary clinic builds on the outcomes of chronic 
venous and capillary disease. The plastic surgery team from Korea used a pulsed dye laser on KTS patients with capillary malformations (port-wine stain), 4 of 19 patients were treated with a laser without any morbidity. The $585 \mathrm{~nm}$ wavelength is said to be effective for treating facial telangiectasia and hemangioma. It is argued that this therapy responds well to light-colored lesions in children, but darker lesions in adults are often unsuccessful and respond better to Crypton and Copper lesions ${ }^{14}$.

Unsuccessful management of varicose veins with non-invasive, sclerotherapy or embolization can be done, whereas imaging of the deep vein system must be done first to avoid postoperative complications and thermal effects. Persistent vein sciatica should be treated with sclerotherapy or embolization. Sclerotherapy produced $90 \%$ good results, but the complication rate ranged from $12 \%$ per therapy and $28 \%$ per patient. $15 \%$ of patients have necrosis of the skin. To reduce complications, increase awareness of injectable agents such as ethanol on the nerves or lesions on the skin. Foam sclerotherapy has also been widely used, Smith reported that 808 patients with CEAP grade 2 underwent injection with ultrasound using diluted sodium tetradecyl, and the result was a decrease in CEAP grade within 6 months of therapy with minimal complications ${ }^{17}$.

In the era of minimally invasive surgery, new techniques in the treatment of vein varicosities such as endovascular radiofrequency ablation or endo venous laser ablation (EVLA) of the persistent embryonic vein or great saphenous vein is currently gaining popularity. Some literature reported the success of radiofrequency procedures and laser thermal ablation in 6 months, 1 year, and 5 years respectively 92\%, 86\%, and $72 \%$. There was no difference between using radiofrequency or laser. Repeat procedures are often performed with minimal complications. If a superficial vein is used, optimal attention should be given, considering that no fever is provided in this procedure, therefore significant burns can occur ${ }^{18}$. The EVLA method is performed by heat-induced collagen shrinkage, followed by fibrotic sealing of vein lumen. Figure 1 showed the reduction of varicosities after EVLA2.

\section{c. Open surgery}

This open surgery therapy has rarely been used in the last few decades. Open surgery is usually targeted at patients who are not candidates for semi-invasive procedures. Subfascial endoscopic perforator surgery is useful in patients with large incompetent venous perforations, but percutaneous intervention is now more frequently used. Ulceration or bleeding can occur, but in KTS patients the wound healing process can be hampered. Therefore, postoperative edema can be relieved by compression and elevation. Prior to intervention, venous anatomy must be documented to avoid destroying the superficial venous system. Because venous damage can occur, the possibility of blood loss during surgery must be considered. Blood transfusions must be taken into account 19 .

Baraldini et al reported 29 pediatric patients with KTS underwent combination open surgery with semiinvasive intervention. The mean age was 10.3 years. Technically successful procedure. No fatal complications or deaths occurred within 6 months to 2 years of follow-up. Intervention at an early age is recommended because it is considered safe and effective in minimizing venous hypertension ${ }^{20}$. The Mayo Clinic group documented the operative experience of KTS patients with radiofrequency and laser ablation and were followed up for 73 months. Most of the patients have complaints of pain. Stripping of the large saphenous vein, small saphenous vein, and lateral embryonic vein was performed. Postoperative morbidity was $4 \%$ having DVT and $14 \%$ thrombophlebitis, and $10 \%$ having at least 1 episode of cellulitis. There were no deaths, nearly $30 \%$ of patients experienced reintervention. However, $50 \%$ of patients reported a significant reduction in pain, and statistically lowered the Venous Clinical Severity Score and CEAP class ${ }^{21}$. 


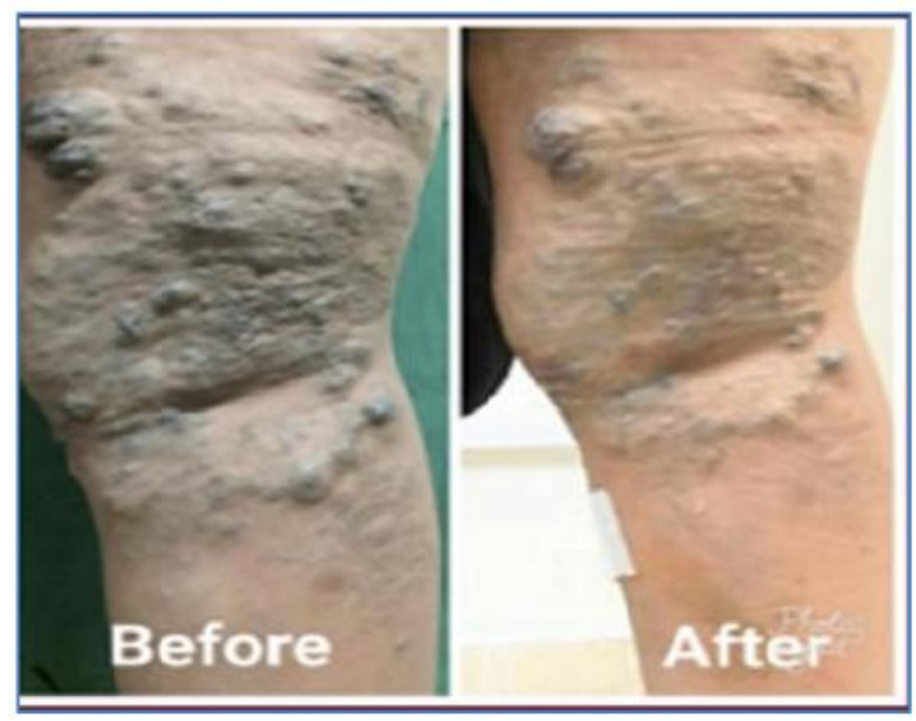

Figure 1. Before and After Endo Venous Laser Ablation Procedure2

\section{Conclusion}

Klippel-Trenaunay syndrome is a rare and complex congenital syndrome that affects both the lower and upper extremities. This syndrome usually occurs sporadically. The three main symptoms of KTS are varicosities and venous malformations, capillary malformations (port-wine stains), and bone and soft tissue hypertrophy. There is no precise pathognomonic test to build the diagnosis of KTS, but determination of the extensiveness and severity of the disease should be considered first. Patients with KTS are generally treated similarly to patients with other chronic venous disorders, starting from non-invasive, semi-invasive, and most invasive or open surgery.

\section{Reference}

1. Wang SK, Drucker NA, Gupta AK, Mashalleck FE, Dalsing MC. Diagnosis and management of the venous malformations of Klippel-Trénaunay syndrome. Journal of Vascular Surgery. 2017;5(4): 587-595.

2. Putra IGAMA, Hidayat NA, Permatananda PANK. Endovascular Laser Ablation: Minimally Invasive Intervention for Klippel-Trenaunay Syndrome. IOP Conf. Series: Materials Science and Engineering 2018; 434:01232.
3. Noel AA, Gloviczki P, Cherry KJ, Rooke TW., Stanson AW, Driscoll DJ. Surgical treatment of venous malformations in Klippel-Trénaunay syndrome. Journal of Vascular Surgery. 2000;32(5):840-848.

4. Purkait R, Samanta T, Sinhamahapatra T, Chatterjee M. Overlap of Sturge-Weber syndrome and Klippel-Trenaunay syndrome. Indian J Dermatol. 2011;56(6):755757.

5. Cha SH, Romeo MA, Neutze JA. Visceral Manifestation of Klippel Trenaunay Syndrome. Radiographics. 2005;25(6): 1941-1946.

6. Sunderkrishnan R. Genetic Predisposition of Klippel-Trenaunay-Weber Syndrome. 2016. (Accessed July 13, 2019, Available at: http://emedicine.medscape.com/article/94576 0-overview).

7. Janniger CK. Klippel Trenaunay Weber syndrome. 2017. (Accessed July 13, 2019, Available at: http: / / reference.medscape.com/article/ 108425 7).

8. Hofer T, Frank J, Itin PH. Klippel-Trenaunay syndrome in a monozygotic male twin: supportive evidence for the concept of 
paradominant inheritance. Eur $J$ Dermatol. 2005; 15(5):341-343.

9. Kihiczak GG, Meine JG, Schwartz RA, Janniger CK. Klippel-Trenaunay syndrome: a multisystem disorder possibly resulting from a pathogenic gene for vascular and tissue overgrowth. Int $\mathrm{J}$ Dermatol. 2006;45(8):883-890.

10. Agale SV, Bharambe BM, Pawar R, Valand AG. Klippel Trenaunay Syndrome: Report of A Rare, Mild Form of Syndrome. Journal of Evolution of Medical and Dental Medicine 2008; 1(5): 693697.

11. Leon CA, Filho LRB, Ferrari MD, Guidolin BL, Maffessoni BJ. Klippel-Trenaunay syndrome Case Report. An. Bras. Dermatol. 2010;85(1): 15.

12. Furness PD, Barqawi AZ, Bisignani G, Decter RM. Klippel-Trenaunay Syndrome: 2 Case Reports and A Review of Genitourinary Manifestations. Journal of Urology. 2001; 166(4):1418-1420.

13. Khilnani NM, Min RJ. Imaging of Venous Insufficiency. Semin. Intervent. Radiol. 2005;22(3):178-184.

14. Sung HM, Chung HY, Lee SJ, Lee JM, Huh S, Lee JW, Choi KY, Yang JD, Cho BC. Clinical experience of the Klippel-Trénaunay syndrome. Arch Plast. Surg. 2015; 42:552-558.

15. Jones RH, Carek PJ. Management of Varicose Veins. American Family Physician. 2008; 78(1): 1289-1294.
16. KTS Working Group. Clinical practice guidelines for Klippel- Trénaunay Syndrome. 2016. (Accessed at July 13, 2019. Available at: https://k-t.org/assets/images/content/BCH-

Klippel- Trenaunay-Syndrome-ManagementGuidelines-1-6-2016.pdf.).

17. Smith PC. Chronic venous disease treated by ultrasound guided foam sclerotherapy. Eur. J. Vasc. Endovasc. Surg. 2006; 32:577-583.

18. Frasier K, Giangola G, Rosen R, Ginat DT. Endovascular radiofrequency ablation: a novel treatment of venous insufficiency in KlippelTrénaunay patients. J. Vasc. Surg. 2008;47: 1339-1345.

19. Ishikawa $\mathrm{K}$, Yamamoto $\mathrm{Y}$, Funayama E, Furukawa H, Sasaki S. Wound Healing Problems Associated with Combined Vascular Malformations in Klippel Trenaunay Syndrome. 2019; 8(6): 246-255.

20. Baraldini V, Coletti M, Cipolat L, Santuari D, Vercellio G. Early surgical management of Klippel-Trénaunay syndrome in childhood can prevent long-term haemodynamic effects of distal venous hypertension. J. Pediatr. Surg. 2002;37:232-235.

21. Malgor RD, Gloviczki P, Fahrni J, Kalra M, Duncan AA, Oderich GS, Vrtiska T, Driscoll D. Surgical treatment of varicose veins and venous malformations in Klippel-Trénaunay syndrome. Phlebology. 2016; 31:209-215. 